\title{
Development of Postural Control in Healthy Children: A Functional Approach
}

\author{
Christine Assaiante, Sophie Mallau, Sébastien Viel, Marianne Jover and Christina Schmitz
}

DPA Group, UMR 6196, CNRS, 31 Chemin Joseph Aiguier 13402 Marseille, Cedex 20 France

\begin{abstract}
SUMMARY
From a set of experimental studies showing how intersegmental coordination develops during childhood in various posturokinetic tasks, we have established a repertoire of equilibrium strategies in the course of ontogenesis. The experimental data demonstrate that the first reference frame used for the organization of balance control during locomotion is the pelvis, especially in young children. Head stabilization during posturokinetic activities, particularly locomotion, constitutes a complex motor skill requiring a long time to develop during childhood. When studying the emergence of postural strategies, it is essential to distinguish between results that can be explained by biomechanical reasons strictly and those reflecting the maturation of the central nervous system (CNS). To address this problem, we have studied our young subjects in situations requiring various types of adaptation. The studies dealing with adaptation of postural strategies aimed at testing short and long-term adaptation capacity of the CNS during imposed transient external biomechanical constraints in healthy children, and during chronic internal constraints in children with skeletal pathologies. In addition to maintenance of balance, another function of posture is to ensure the orientation of
\end{abstract}

Reprint requests to: Christine Assaiante, DPA Group, UMR 6196,CNRS, 31 Chemin Joseph Aiguier 13402 Marseille, Cedex 20 France; e-mail: christine.assaiante@ dpm.cnrs-mrs.fr

O 2005 Freund \& Pettman, U.K. a body segment. It appears that the control of orientation and the control of balance both require the trunk as an initial reference frame involving a development from egocentric to exocentric postural control. It is concluded that the first step for children consists in building a repertoire of postural strategies, and the second step consists in learning to select the most appropriate postural strategy, depending on the ability to anticipate the consequence of the movement in order to maintain balance control and the efficiency of the task.

\section{KEYWORDS}

human development, balance strategies, segmental stabilization, orientation, adaptation

\section{INTRODUCTION}

Postural control is integral to the execution of goal-directed action. The most important function of posture is to ensure the maintenance of equilibrium during the initiation and continuation of movement. In addition, posture serves as a reference frame for the production of accurate movements. Therefore, postural strategies have to be adapted to various contexts and environments. Our contribution to this field lies in a functional approach to motor development. This functional approach involves a gradual mastering of coordination, anticipation, and adaptation in postural control in the course of ontogenesis, from babies 
to teenagers (Assaiante, 2000).

According to their ontogenetic model of balance control, Assaiante and Amblard (1995) assumed that the various balance strategies adopted by children, as well as by adults, involve taking into account two main functional principles of spatial organization. The first concerns the choice of the stable reference frame on which the equilibrium control is based, and the second concerns the gradual mastery of the degrees of freedom of the various body joints. The choice of the stabilized anatomical segment of reference, as well as the character of coupling between articulations, depends on (a) the dynamic constraints determining the difficulty of a motor task, (b) the environment, and (c) the characteristics of each developmental period.

In many posturokinetic activities, the contact with the support is intermittent. During locomotion, for example, the lower limbs cannot serve as a stable reference frame for the organization of the whole-body balance. Thus, it is necessary to stabilize, at least one anatomical segment, which then constitutes the reference value around which movements can be built up. The reference frame can be either the pelvis to allow a better control of the center of gravity or the head to allow clear vision and better visual and vestibular processing, or both segments, according to the difficulty of the task. Moreover, human stance involves superimposed modules from the feet to the head, each with its own specific central and peripheral regulation, which can be controlled more or less independently (Gurkinkel et al., 1971; Massion, 1992). Classically, two modes of control are possible. The "en bloc" strategy consists of minimizing the number of degrees of freedom to be controlled simultaneously during the movement, according to Bernstein's theory (1967). The "articulated" strategy consists of controlling independently a couple of consecutive anatomical segments and requires the mastery of the degrees of freedom of the corresponding joint.
The various balance strategies also involve taking into account two main functional principles of temporal organization (Assaiante \& Amblard, 1995). These authors assumed that the stabilized anatomical segment constitutes the origin of the temporal organization of balance control. Segments are involved in movement in an ascending or descending sequence, depending upon the anatomical segment that serves as the reference frame. For example, on a standing task on a stable support surface, the balance control is organized from the feet to the head, in ascending order. In contrast, in a more dynamic situation, as walking on a narrow beam, the stabilized reference frame can be the head, as it is the case in adults and in children from 7 years of age onward (Assaiante \& Amblard, 1993). Balance control is thus organized from the head to the feet, in descending order. In addition, this multi-segmental control also implies an efficient coordination between posture and movement that can be organized in a feed-forward or a feed-back mode.

\section{BUILDING A REPERTOIRE OF BALANCE STRATEGIES}

The early stage of independent walking in toddlers offers an excellent opportunity for equilibrium studies. Maintaining balance during locomotion is a complex task because it involves achieving a compromise between the forward propulsion of the body, which is a highly destabilizing force, and the need to maintain the lateral stability of the body. Even simple walking on a flat surface, free of obstacles, poses a considerable balance problem to young walkers. Indeed, the difficulty of maintaining equilibrium during locomotion is further accentuated by the fact that the weight of the whole body must be supported by one leg at a time during the swing phase of gait. This is the most difficult balance problem encountered by infants learning to walk 
(Assaiante, 1998; Assaiante \& Amblard, 1993; Brenière et al., 1989; Thelen, 1984).

We have studied the lateral balance control of the upper body segments during locomotion, in infants from 1 week to 11 months of walking experience (Assaiante, 1998; Assaiante \& Amblard, 1993). The first result to be noted is that pelvis stabilization in space strategy is efficiently used as soon as autonomous walking appears and might be a prerequisite for the emergence of independent walking. Secondly, the shoulder stabilization in space appears only at the second month of autonomous walking. Finally, no preferred head stabilization, neither in space nor on the shoulders, has yet appeared at the eleventh month of autonomous walking. The results clearly indicate an ascending progression with age of the ability to control the upper body segments during locomotion.

Figure 1 summarizes the temporal organization of balance control in young walkers. A calculation of the inter-correlation functions has revealed that the movement of shoulders precedes that of the head and that the movement of the pelvis precedes that of the shoulders, suggesting an ascending temporal organization from the pelvis to the head. Moreover, EMG recordings report that the pelvis is stabilized before foot lift-off, suggesting a descending temporal organization of balance control from pelvis to feet. Therefore, at this early age, rather than a simple temporal ascending organization, there is a pelvis-centered organization of balance control during locomotion. The pelvis constitutes thus the first stable reference frame, around which locomotor balance control can be built up.

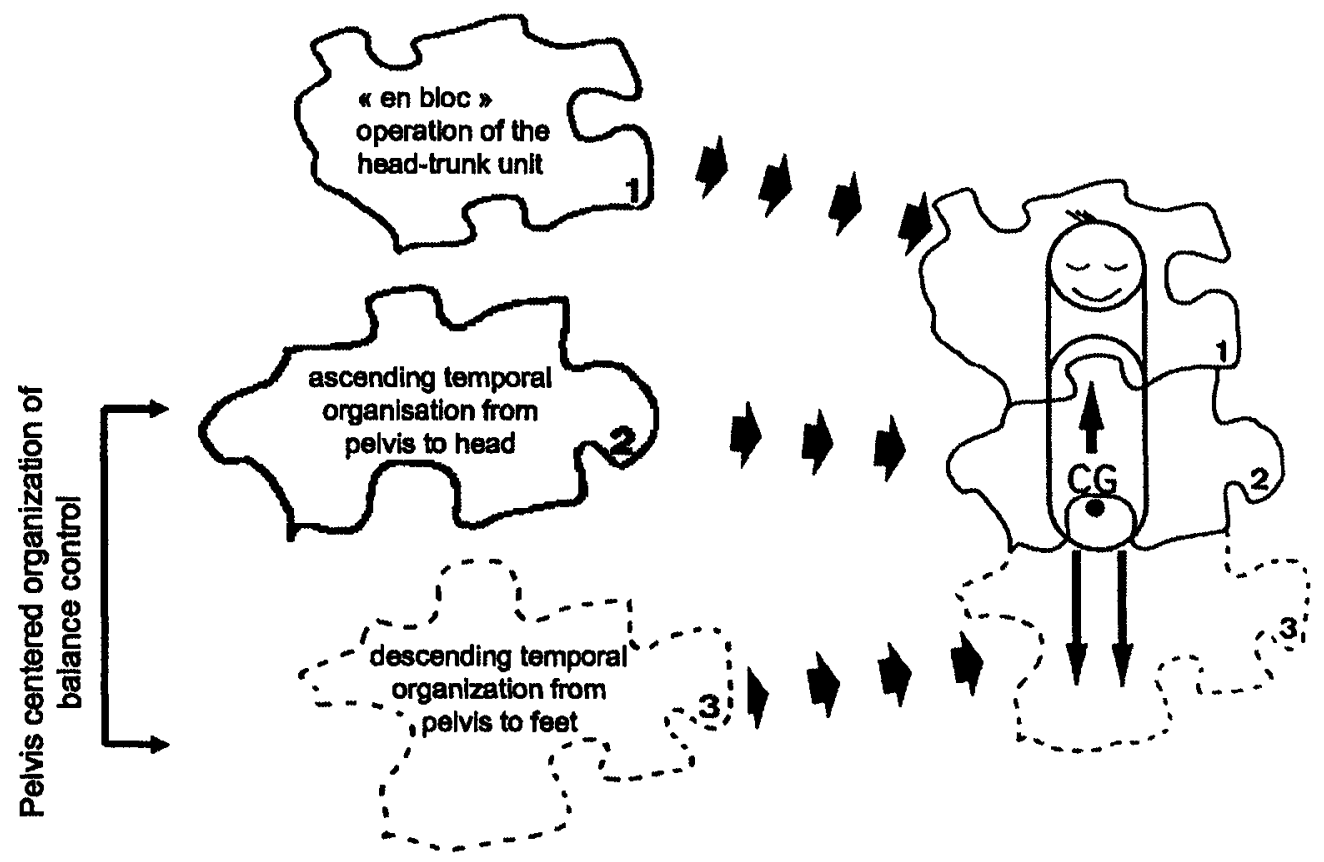

Fig. 1: Scheme of the organization of balance control in toddlers 
In the literature, many authors, such as Berthoz and Pozzo (1988), Pozzo et al. (1990), Grossman et al. (1988; 1989), Ripoll et al. (1986), Assaiante and Amblard (1993), have demonstrated that in adults the head is stabilized in space in various posturokinetic activities, presumably to allow better visual fixation and better vestibular processing, as well as to provide a stable reference frame around which movements can be built up. During childhood, controlling head stabilization during locomotor activities constitutes a complex motor skill that takes a long time to mature (Assaiante \& Amblard, 1995). In a previous developmental study, Assaiante and Amblard (1993) investigated the development of head control during various locomotor tasks. In fact, it is possible to discern at least three main developmental phases.

The first phase includes children from 3 to 6 years of age, who adopt head stabilization in space strategy only while walking on the flat ground without any equilibrium difficulty. When the level of equilibrium difficulty increases, these children show an increase in the head-trunk stiffness, particularly in 6-year-old children. This suggests an en bloc operation of the head-trunk unit.

The second phase includes children from 7 to 8 , who become able to adopt the head stabilization in space strategy even when balance difficulty increases, for example while walking on narrow supports. This improvement is associated with a large decrease in the correlations calculated between the head and the trunk movements of rotation, consistent with an articulated operation of the head-trunk unit.

Lastly, in adulthood, the head stabilization in space strategy is adopted most of the time, but only in the case of the roll, which is the most relevant component of rotation to control the lateral body oscillations while walking. Moreover, the development of head control is not linear. In fact, the development of the head stabilization in space displays a transition phase between 6 and 7 years of age. In 6-year-old children, the beginning of a systematic use of the head stabilization in space strategy observed in 7-year-old children walking on narrow supports was preceded by a sort of regression to adopt the alternative head stabilization on trunk strategy.

\section{SHORT AND LONG TERM POSTURAL ADAPTATION}

When studying the emergence of postural strategies, it is essential to distinguish between results due to biomechanical reasons strictly and those reflecting the maturation of the CNS. To address this problem, we have studied various types of postural adaptation based either on specific constraints given by the character of the task that covered situations of various balance difficulty or on transitory biomechanical constraints, such as using a splint blocking one knee to destabilize the pelvis; or on chronic skeletal abnormalities, such as torsion of the lower extremities (Assaiante, 2000).

Figure 2 summarizes the main adaptive strategies, in terms of segmental stabilization in the frontal plane and corresponding temporal organization. 3- to 4-year-old children are able to stabilize pelvis and shoulders in space when walking on flat ground. Increasing the task difficulty by walking on a narrow line leads to a loss of shoulder stabilization, which can, however, be re-established if the pelvis is destabilized. Early head stabilization in space, as a substitution for the destabilized pelvis that constitutes the first reference frame, does not occur, contrary to our original hypothesis. Thus, short-term adaptation strategies are selected strictly from the repertoire already present at the studied age. The temporal organization is ascending from the stabilized pelvis to the head. If the pelvis is destabilized, the shoulders tend to become the origin of the temporal sequence. 


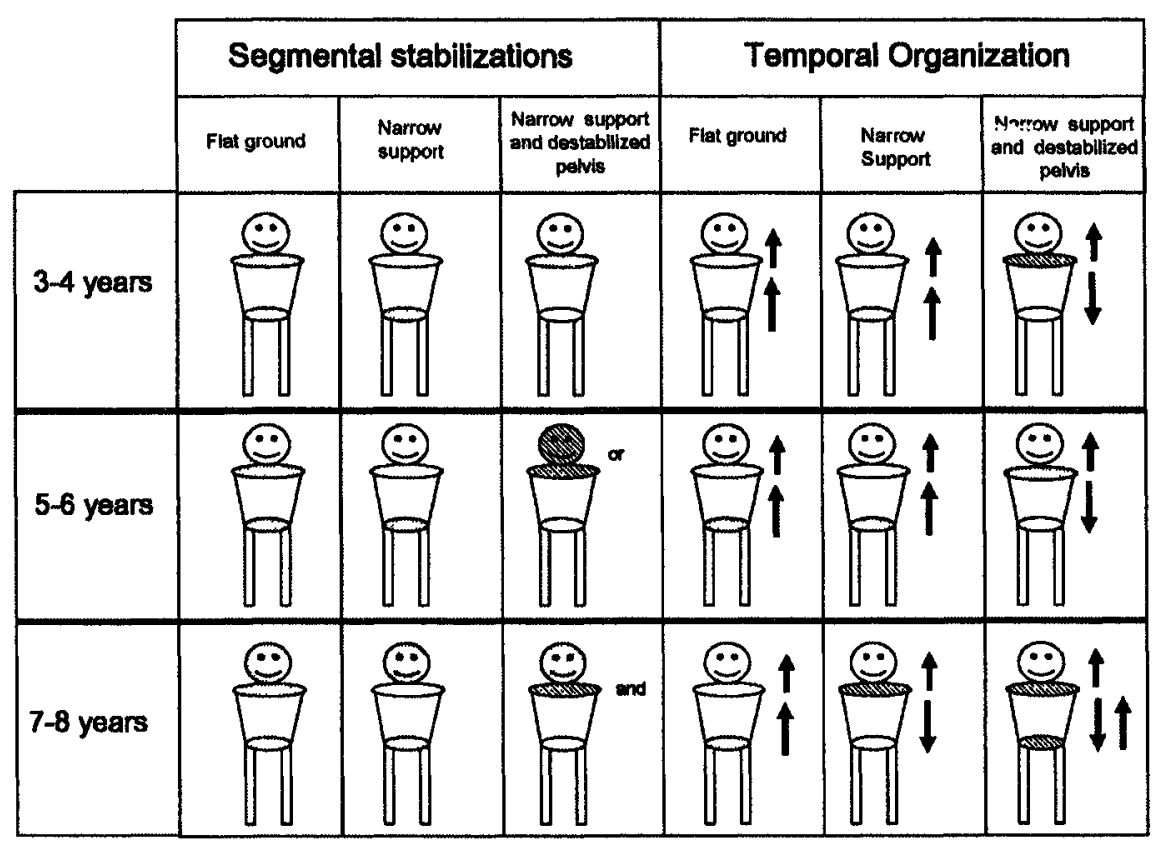

Fig. 2: Main adaptive strategies according to the age and the increasing difficulty of equilibrium task. The range goes from simple walking on flat ground to walking on a line at imposed speed by means of the treadmill.

When walking on a flat ground or a beam, 5to 6-year-old children show the same stabilization patterns as the younger group does. On the other hand, with a destabilized pelvis, no systematic shoulder stabilization in space is present, and the head has not yet acquired this ability either. The results confirm that at around the age of 6 , a turning point appears in the development of equilibrium control, as already reported (Assaiante \& Amblard, $1993 ; 1995)$. The temporal organization is practically identical to that of the 3- to 4-year-old children, with the exception of the most difficult situations, when the temporal sequence starts from the shoulders. In other words, the temporal organization of balance control is shoulder-centered.

Finally, 7- to 8-year-old children stabilize the pelvis, the shoulders, and the head, suggesting an independent control of each anatomical segment while walking on a flat surface. When balance difficulty increases, for example during walking on a narrow surface, the shoulder stabilization disappears. In the most difficult situation with the imposed pelvis destabilization, the head remains stabilized in space, as do the shoulders sometimes. Under the easiest conditions, the temporal organization of the movement is still centered on the pelvis, like in younger children. When walking along a line, the time sequence starts at the shoulders. In contrast, with destabilized pelvis, where one would expect a descending mode of temporal organization, the temporal pattern is completely erroneous. In fact, we observed a sort of regression in the temporal organization of balance control that just precedes the establishment of the descending 
temporal organization from the head stabilized to the feet. The latter results of the 7- to 8-year group remind us that even though segmental stabilization and temporal organization usually go hand in hand, in certain developmental periods it is important to emphasize the time-lag between the mastery of the segmental stabilization that occurs first and the mastery of the corresponding temporal organization that occurs in a second time. Various developmental studies in posture (Eliasson et al., 1995; Konczak \& Dichgans, 1997; Schmitz et al., 1999; 2002) also reported that during childhood the control of timing seems to be the most difficult parameter to acquire.

In a recent study, we investigated the longterm adaptability of the CNS in controlling upper body segments during various locomotor tasks in 5- to 6-year-old and 7- to 10-year-old children with internal rotations (IR) of the lower limbs and free from neurological dysfunction (Mallau et al., submitted). Their in-toeing gait results either from a persistent femoral torsion or from an internal tibial torsion. All these children, whatever their age, showed a lower gait velocity, particularly in difficult balance conditions, associated with a decrease of yaw and roll shoulder stabilization in space. However, in 5-to 6-year-old children, the effect of the local biomechanical deficit remained limited to the lower limbs and did not affect the upper body coordination. By contrast, in the 7-to 10-year-old children, the development of head stabilization in space was affected, as demonstrated by an en bloc operation of the head-trunk unit instead of the articulated mode of operation of the head-trunk unit systematically adopted by the control group. Thus, as pelvis stabilization remains the main reference frame to organize balance control in older children, the biomechanical anomaly of the legs causes an alternative postural strategy, with respect to control children. Further investigations in teenagers should help to test if these skeletal abnormalities of the lower extremities only delay the use of the head reference frame or if they remain a life-long obstacle to the building of the repertoire of reference frames.

\section{DEVELOPMENT OF POSTURAL ORIENTATION}

In addition to the maintenance of balance and segmental stabilization, another function of posture is to ensure global body orientation and segmental orientation. The trunk is a key segment to organize postural stabilization and orientation control (Massion, 1998). In a previous developmental study we tested the ability of children to maintain a horizontal forearm position in various tasks involving either voluntary or involuntary trunk motion (Assaiante, 2000; Roncesvalles et al., in press).

We simply suggested to our $2-3$ year olds to blow bubbles (Fig. 3). In the starting position, the child was holding a tube full of liquid without any problem. The forearm was horizontal and the trunk vertical. In the next step, however, we asked her to take a deep breath and blow bubbles. When she leans her trunk forward, she spills the liquid, as a relatively constant angle is maintained between the forearm and the trunk. This observation led us to conclude that until the age of 3 years, the major postural reference for limb orientation control is the trunk, which is consistent with an egocentric control of orientation.

The protocol was modified for older children, but the question concerning the coupling between the trunk and the forearm remained the same. The children were sitting in the same position, with their left forearm supporting a tray on which a cup was posed. Contrary to the experiment described above, the trunk was not perturbed by voluntary inclination but rather by sinusoidal antero-posterior oscillations of the platform on which the subjects were sitting. However, the repetition of the cycle of oscillations enabled the subjects to anticipate the perturbation and to stabilize their forearm in a horizontal position, as in voluntary leaning. We 

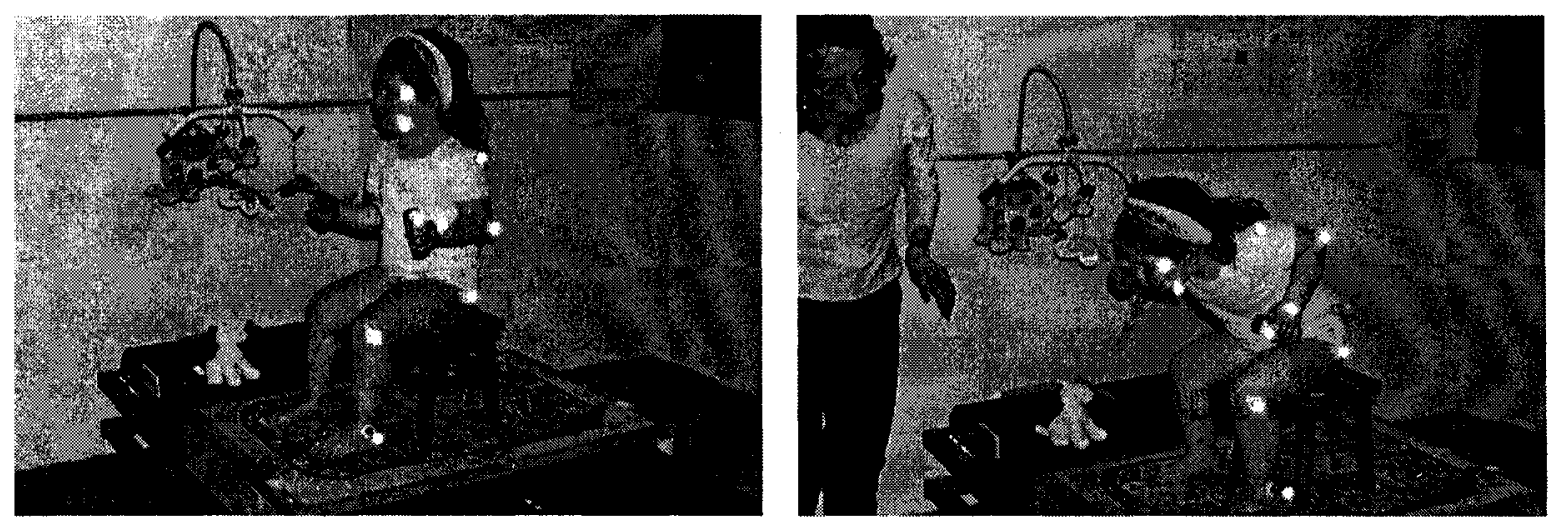

Fig. 3: A 2-year-old child prepares to blow bubbles (left photo) then leans forward to complete the task (right photo). The photo shows the child's use of an egocentric rather than an exocentric reference frame, as she maintains a relatively constant angle between the forearm and the trunk. Little effort is made to orient the forearm (and bottle) to the vertical line of gravity.

calculated an anchoring index between the forearm and trunk angular dispersions (Assaiante, 2000).

The positive values of anchoring index in adults as in 7 - to 10 -year-old children show that the forearm was stabilized in space rather than on the trunk, which means that the movements of these two segments are independent. In contrast, children aged 4 to 6 years do not show a significant positive anchoring index. This result suggests that younger children show much stronger forearm-trunk coupling than older children do. The results again evoke a transition between 6 and 7 years from a more global postural control to a selective control of independent body segments and from an ego-centric to exocentric control of orientation.

During ontogenesis, periods of relatively stable increase in body size alternate with periods of accelerating growth. Puberty is characterized by important morphological and functional changes during a short time (Keogh \& Sugden, 1985). Moreover, the body scheme, slowly built during childhood by the integration between vestibular, visual, and somatosensory information, is probably affected during puberty. The body scheme contributes to the development of the internal representation of action that constitutes the base of feed-forward control to compensate in advance for the destabilizing effects of the movement. In a recent study, we asked a question about the consequences of the changes that occur during puberty for postural control and which sensory-motor strategies do teenagers use to preserve their postural performance (Viel, 2003; Viel et al., unpublished).

To answer to these questions, 14- to 15-yearold teenagers $(n=20)$ were asked to maintain their vertical body orientation despite very slow lateral oscillations of the support, with or without vision in a standing position. The imposed oscillations of the support were chosen in amplitude and frequency to be either above $\left( \pm 5^{\circ}\right.$ at $\left.0.06 \mathrm{~Hz}\right)$ or below $\left( \pm 5^{\circ}\right.$ at $0.001 \mathrm{~Hz}$ ), the semicircular canal threshold. Thus, the lower frequency of the support associated with eyes closed provides an excellent opportunity to investigate selectively the proprioceptive contribution to postural control in teenagers. 


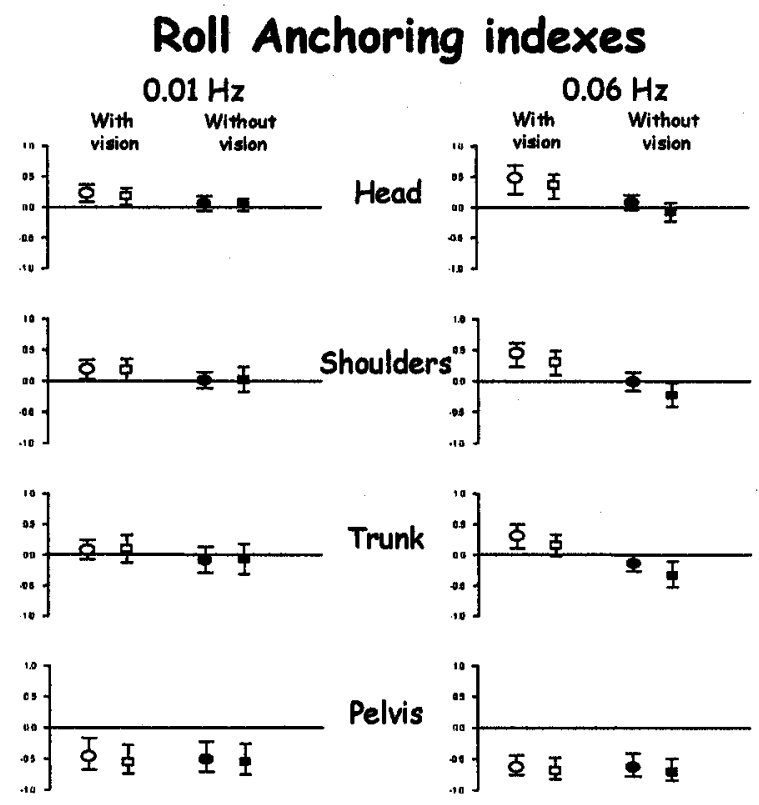

Fig. 4: Mean anchoring indexes and standard deviation of head, shoulders, trunk and pelvis with vision (in white circle for girls and in white square for boys) and without vision (in black circle for girls and in black square for boys) in the case of lateral perturbation of the support. Grey squares represent adults without vision.

The roll-anchoring indexes presented in Fig. 4 reveal that teenagers differ from young adults concerning the stabilization in space strategies for both frequencies. Most of the time, the values of the anchoring indexes obtained in adults are positive, indicating an independent control of the segment with respect to the support's perturbation. Moreover, young adults show a pelvis-stabilization in space strategy, whereas teenagers show a pelvisstabilization on support strategy, which is consistent with an en-bloc postural strategy, mainly selected without vision. Based on this study, the stabilization in space strategies appear to improve significantly from teenagers to young adults, from an en bloc to articulated operations of the headtrunk unit.

Lastly, teenagers seem more dependent on visual cues than are adults, which is consistent with a transient proprioceptive neglect hypothesis in sensory integration for postural control.

\section{CONCLUSION}

In conclusion, the overall results presented in this paper support the concept of multiple reference frames: stabilization of the head or stabilization of the pelvis, which operate in a complementary manner or in concert, associated with an en bloc or articulated operation of the body joint to permit the most appropriate temporal organization of balance control during action. The first step for children consists of building a repertoire of postural strategies. The second step consists of learning to select the most appropriate postural strategy depending on the ability to anticipate on the consequence of the movement in order to maintain balance control and the efficiency of the task. Developmental studies involving postural control during various posturokinetic tasks suggest that anticipatory control, despite its early emergence, slowly matures during childhood (Haas et al, 1989; Hay \& Redon, 1999; 
2001, Assaiante et al, 2000), as well as the mastering of timing parameters (Eliasson et al., 1995; Konczak and Dichgans, 1997; Schmitz et al., 1999; 2002). Precise mastering of timing parameters seems to be one of the key factors of the anticipatory function that reflects the maturation of the CNS. Taking into account the complexity of the parameters to control, it is not surprising that the development of postural control continues up to late periods during childhood and adolescence. The recent development of fMRI studies should help us to better understand the relations between the late maturational process of the CNS and the late development of postural control, particularly at the periods of transition such as 6-7 years and adolescence.

\section{ACKNOWLEDGMENTS}

We are grateful to the children who participated in these studies and their parents. We wish to thank Bernard Amblard for his helpful comments and for critically reviewing the manuscript. This work was supported by grants from the Centre National de la Recherche Scientifique (CNRS) and l'Assistance Publique des Hôpitaux de Marseille (APHM).

\section{REFERENCES}

Assaiante C. 1998. Development of locomotor balance control in healthy children. Neurosci Biobehav Rev 1998; 22: 527-532.

Assaiante C. 2000. Construction du repertoire des référentiels posturaux: maturation et adaptation au cours de l'enfance. Habilitation à Diriger des Recherches en Neurosciences: Mediterranean University, France.

Assaiante C, Amblard B. 1993. Ontogenesis of head stabilization in space during iucomotion in children: influence of visual cues. Exp Brain Res 93: 499-515.

Assaiante C, Amblard B. 1995. An ontogenetic model for the sensori motor organization of balance control in humans. Hum Mov Sci 14: 13-43.
Assaiante C, Woollacott MH, Amblard B. 2000. Development of postural adjustment during gait initiation: Kinematic and EMG analysis. $\mathrm{J}$ Mot Behav 32: 211-26.

Bernstein NA. 1967. The coordination and regulation of movements, Oxford, UK: Pergamon Press.

Berthoz A, Pozzo T. 1988. Intermittent head stabilization during postural and locomotory tasks in humans. In: Amblard B, Berthoz A, Clarac F, eds, Posture and Gait : Development, Adaptation and Modulation. Amsterdam, the Netherlands: Elsevier Science, 1988. 189-198.

Brenière Y, Bril B, Fontaine R. 1989. Analysis of the transition from upright stance to steady state locomotion in children with under 200 days of autonomous walking. J Mot Behav 1989. 21: 20-37.

Eliasson A-C, Forssberg H, Ikuta K, Apel I, Westling G, Johansson RS. 1995. Development of human precision grip. V. Anticipatory and triggered grip actions during sudden loading. Exp Brain Res 106: 425-33.

Grossman GE, Leigh RJ, Abel LA, Lanska DJ, Thurston SE. 1988. Frequency and velocity of rotational head perturbations during locomotion. Exp Brain Res 70: 470-476.

Grossman GE, Leigh RJ, Bruce EN, Huebner WP, Lanska DJ. 1989. Performance of the human vestibuloocular reflex during locomotion. J Neurophysiol 62: 264-272.

Gurkinkel VS, Kots Y, Paltsev Y, Feldman A. 1971. The compensation of respiratory disturbances of the erect posture of man as an exemple of the organiszation of interarticular interaction. In: Gelfand J, Gurkinkel, VS, Fomin S, Tsetlin M, eds, Models of the Structural-Functional Organization of Certain Biological Systems. Cambridge, Massachusetts, USA: MIT Press; 382-395.

Haas G, Diener HC, Rapp H, Dichgans J. 1989. Development of feedback and feedforward control of upright stance. Dev Med Child Neurol 31: 481-488.

Hay L, Redon C. 1999. Feedforward versus feedback control in children and adults subjected to a postural disturbance. Exp Brain Res 125: 153-62.

Hay L, Redon C. 2001. Development of postural adaptation to arm raising. Exp Brain Res 139: 224-232.

Keogh J, Sugden D. 1985. Movement Skill Development, New York, NY, USA: Macmillan 1985.

Konczak J, Dichgans J. 1997. The development toward stereotypic arm kinematics during reaching in the 
first 3 years of life. Exp Brain Res 117: 346-354.

Massion J. 1992. Movement, posture and equilibrium: Interaction and coordination. Progr Neurobio 38: $35-56$.

Massion J. 1998. Postural control systems in developmental perspective. Neurosci Biobehav Rev 22: 465-472.

Pozzo T, Berthoz A, Lefort L. 1990. Head stabilization during various locomotor tasks in humans. I. Normal subjects. Exp Brain Res 82: 97-106.

Ripoll H, Bard C, Paillard J. 1986. Stabilization of head and eyes on target as a factor in successful basketball shooting. Hum Mov Sci 1986. 5: 47-58.

Roncesvalles N, Schmitz C, Zedka M, Assaiante C, Woollacott $\mathrm{MH}$. In press. From egocentric to exocentric spatial orientation development of postural control in bimanual and trunk inclination tasks. J Mot Behav
Schmitz C, Martin N, Assaiante C. 1999. Development of anticipatory postural adjustments in a bimanual load-lifting task in children. Exp Brain Res 126: 200-204.

Schmitz C, Martin N, Assaiante C. 2002. Building anticipatory postural adjustment during childhood: a kinematic and electromyographic analysis of unloading in children from 4 to 8 years of age. Exp Brain Res 2002. 142: 354-364.

Thelen E. 1984. Learning to walk: ecological demands and phylogenetic constraints. In: Lipsitt LP, ed, Advances in Infancy Research, Volume III. Norwood, New Jersey, USA: Ablex; 213-250.

Viel S. 2003. Evaluation du contrôle postural au cours d'oscillations lentes du support chez des adolescents de 14-15 ans. Mémoire de Diplôme d'Etudes Approfondies: Université de Bourgogne, France 

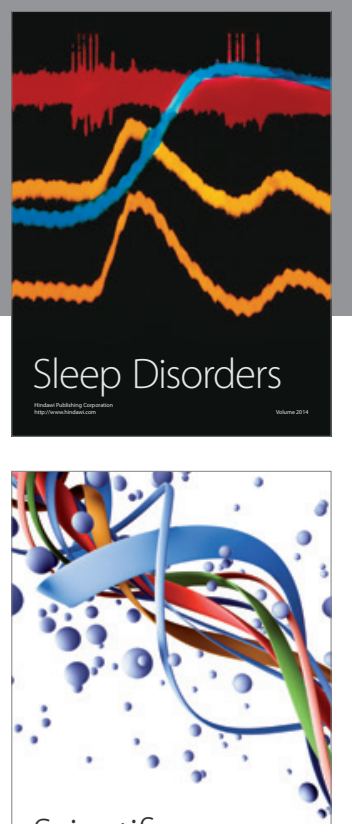

Scientifica
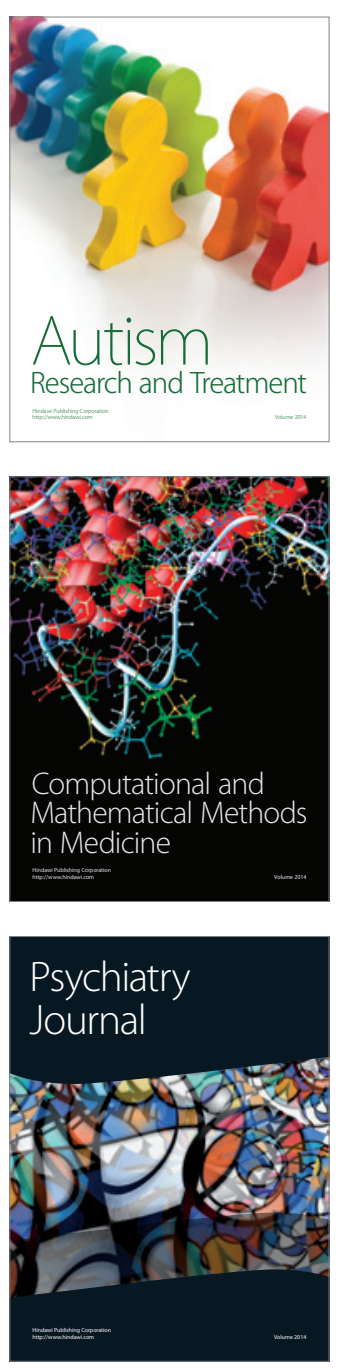
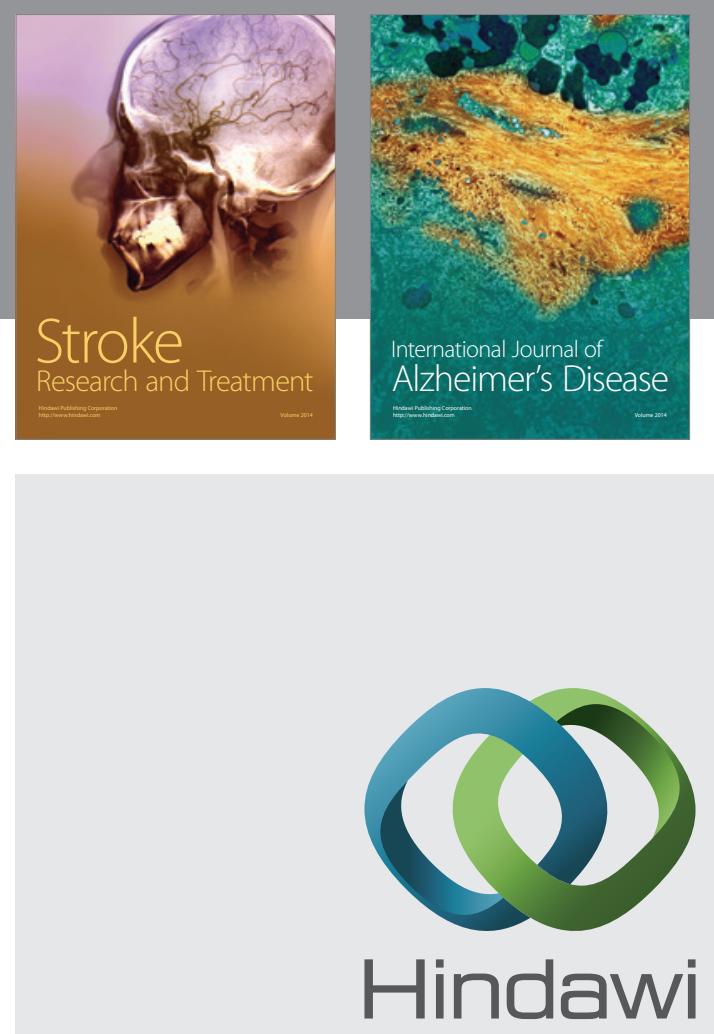

Submit your manuscripts at

http://www.hindawi.com
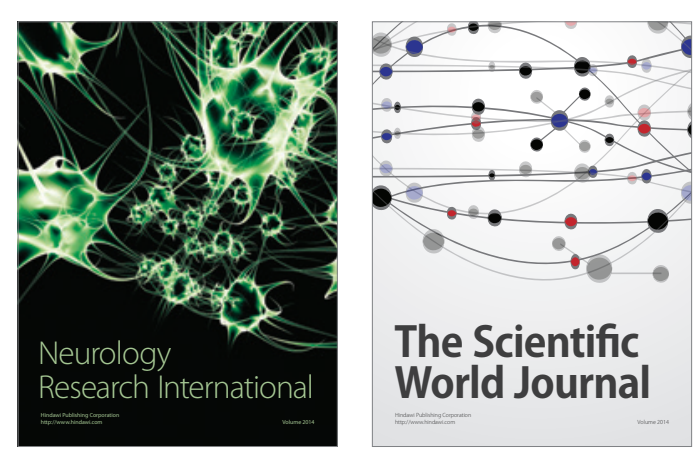

The Scientific World Journal

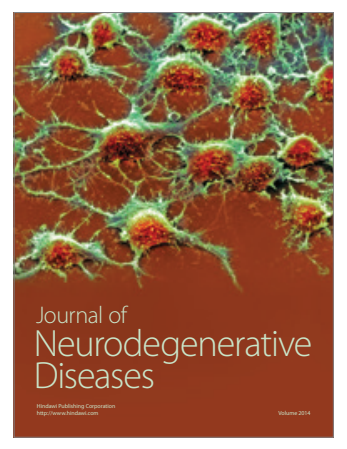

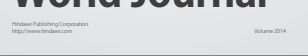

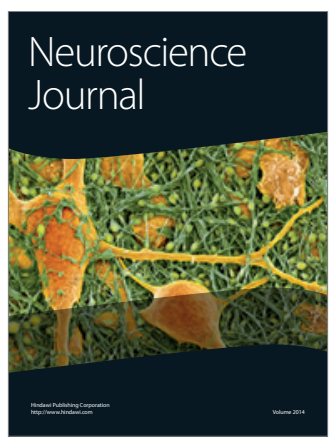

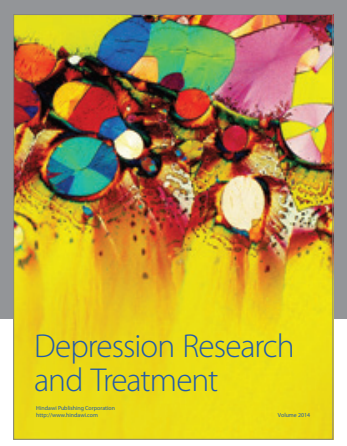
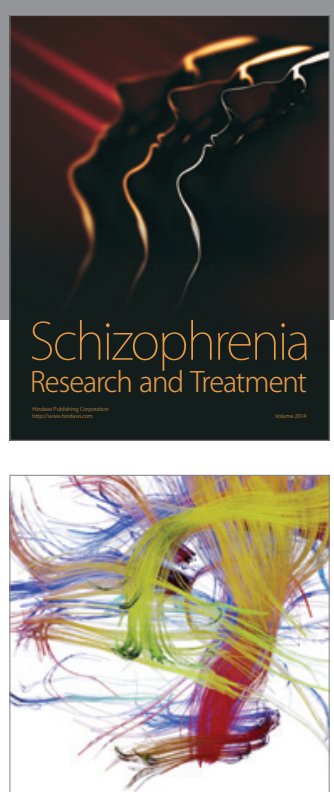

Brain Science

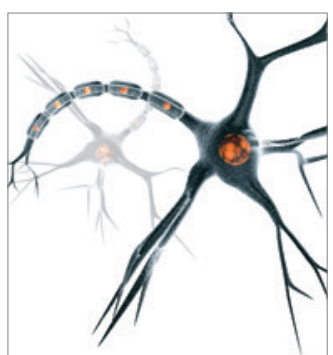

Neural Plasticity
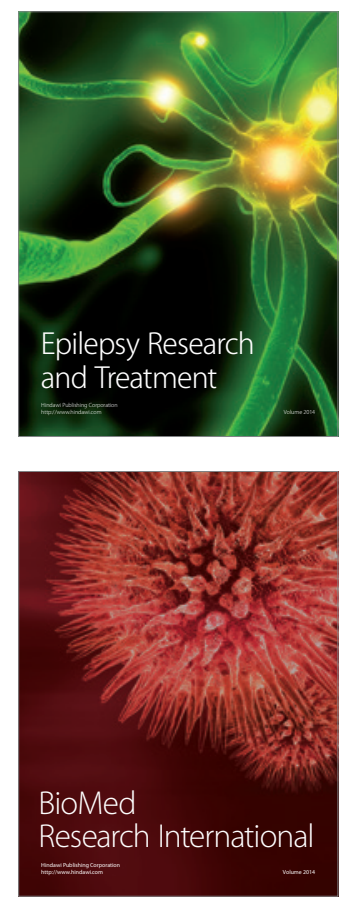

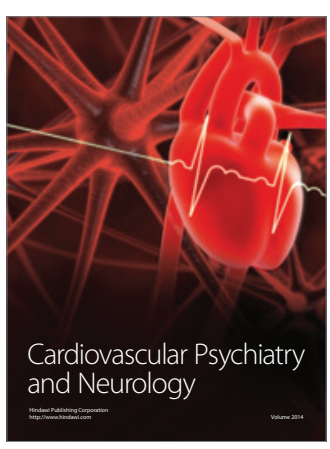

Parkinson's

Disease
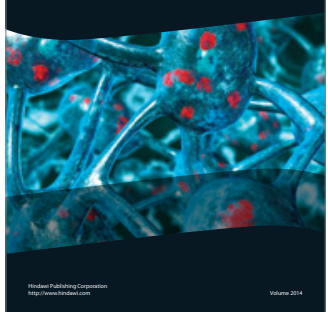